\title{
Assessment of situation awareness during robotic-assisted surgery using multimodal data
}

\author{
Aurelien Lechappe ${ }^{1,2,4}$, Mathieu Chollet ${ }^{1,2}$, Jerome Rigaud ${ }^{3}$, Caroline G.L. Cao ${ }^{1,2,5}$ \\ ${ }^{1}$ IMT Atlantique Bretagne-Pays de la Loire, ${ }^{2}$ Laboratoire des Sciences du Numerique de Nantes (LS2N), ${ }^{3}$ Centre \\ Hospitalier Universitaire de Nantes, ${ }^{4}$ Université de Bretagne Occidentale (UBO), ${ }^{5}$ Wright State University
}

\begin{abstract}
The use of robotic surgical systems disrupts existing team dynamics inside operating rooms and constitutes a major challenge for the development of crucial non-technical skills such as situation awareness (SA). Techniques for assessing SA mostly rely on subjective assessments and questionnaires; few leverage multimodal measures combining physiological, behavioural, and subjective indicators. We propose a conceptual model relating SA with mental workload, stress and communication, supported by measurable behaviours and physiological signals. To validate this model, we collect subjective, behavioural, and physiological data from surgical teams performing radical prostatectomy using robotic surgical systems. Statistical analyses will be performed to establish relationships between SA, subjective assessment of stress and mental workload, communication processes, and the surgeons' physiological signals.
\end{abstract}

\section{CCS CONCEPTS}

- Human-centered computing $\rightarrow$ Collaborative interaction.

\section{KEYWORDS}

Mental Workload; Stress; Communication; Physiological Signals

\section{ACM Reference Format:}

Aurelien Lechappe ${ }^{1,2,4}$, Mathieu Chollet ${ }^{1,2}$, Jerome Rigaud ${ }^{3}$, Caroline G.L. $\mathrm{Cao}^{1,2,5} .2020$. Assessment of situation awareness during robotic-assisted surgery using multimodal data. In Companion Publication of the 2020 International Conference on Multimodal Interaction (ICMI'20 Companion), October 25-29, 2020, Virtual event, Netherlands. ACM, New York, NY, USA, 5 pages. https://doi.org/10.1145/3395035.3425205

\section{INTRODUCTION}

Many laparoscopic surgical procedures are increasingly performed with a robotic surgical system. From the robotic console, the surgeon controls the movement of the surgical tools with high precision and accuracy (Figure 1). Robotic-assisted surgery (RAS) has many advantages for both patients and surgeons. However, new challenges are created by the use of this device such as the need to reorganize the operating room layout and the location of the surgical team around the patient. These changes create a new team dynamic inside the operating room. For instance, communication

Permission to make digital or hard copies of all or part of this work for personal or classroom use is granted without fee provided that copies are not made or distributed for profit or commercial advantage and that copies bear this notice and the full citation on the first page. Copyrights for components of this work owned by others than ACM must be honored. Abstracting with credit is permitted. To copy otherwise, or republish, to post on servers or to redistribute to lists, requires prior specific permission and/or a fee. Request permissions from permissions@acm.org.

ICMI '20 Companion, October 25-29, 2020, Virtual event, Netherlands

(C) 2020 Association for Computing Machinery.

ACM ISBN 978-1-4503-8002-7/20/10 . \$15.00

https://doi.org/10.1145/3395035.3425205 amongst team members is altered, relying mostly on verbalization due to the physical barrier imposed by the robotic system.

The development of non-technical skills (NTS) is necessary for surgery to run smoothly, as technical skills alone are not sufficient to guarantee patient safety and positive outcomes. In surgery, a lack of communication and other NTS has been linked to a higher risk of surgical complications [29, 43]. Non-technical skills are composed of cognitive skills, social skills and operator's personal resources [2]. Cognitive skills includes decision making and situation awareness (SA). Social skills encompass communication, teamwork, and leadership. Personal resources refer to factors such as fatigue and stress [2]. According to Pradarelli et al., SA is considered as the most important of non-technical skills since other NTS are dependent on its development [29]. However, in dynamic and complex environments, such as in surgery, where large amounts of information is available and needed to perform the task, developing and maintaining SA is a particularly complex task [14].

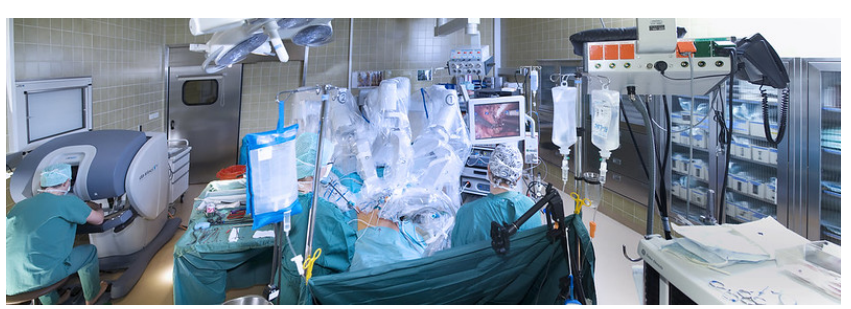

Figure 1: Da Vinci robotic surgical system. @Ars Electronica

In this paper, we introduce a framework for understanding and eventually assessing SA in RAS contexts. We model SA and its relationship with measurable physical and cognitive parameters, such as communication, heart rate, skin conductance, etc., and describe our ongoing work to validate this model in the wild.

\section{THEORETICAL BACKGROUND}

\subsection{Definition of Situation Awareness}

Endsley defines SA in a general context as "The perception of the elements in the environment within a volume of time and space, the comprehension of their meaning, and the projection of their status in the near future" [10](p.36). This breaks down SA into three distinct levels: (1) perception of the environment; (2) understanding the meaning of the perceived elements; (3) and projection of the future status of the environment's elements. The development of the third level requires that the operator has first reached SA levels one and two. In surgery, a similar three-layer with definition of SA has been proposed: "developing and maintaining a dynamic awareness of the situation in the operating room, based on assembling data from the environment (patient, team, time, displays, equipment), 
understanding what they mean, and thinking ahead about what may happen next" [42].

Smith and Hancock [37] describe SA as an "adaptive, externally directed consciousness directly related to stress, mental workload, and other energetic constructs that are facets of consciousness" (p.138). Therefore, the influence of other cognitive factors have to be considered for assessing SA. Further, Salas et al. [33] introduce the concept of team situation awareness. This concept was defined as the aggregation of all individual SA and shared mental models at one point in time, by means of team processes [5]. SA should thus be considered both at the individual and team levels.

\subsection{Assessment of situation awareness}

Several techniques have been developed to assess SA [1]. One such technique, called « Freeze-probe technique » requires the operator to pause during a task to answer questions about the situation. Endsley's SAGAT survey [7] is the most commonly used instrument for this and has been applied in the medical area [14]. The "Real-time probe technique" is similar except that the questions are administered during the action without pausing. SA can also be assessed using self-rating techniques. The most commonly used instrument is Selcon et al.'s SART [35].

Less intrusive techniques for assessing SA include observations by experts [1]. Some surgical non-technical skills observation scales focus on assessing an individual operator (e.g. NOTSS [42], ICARS [31]), while others assess each team member and then derive an aggregated score of the team' skills from individual assessments (e.g. OTAS [16], SPLINTS [26]). Performance measures can also be used to assess SA:different assessment methods are described by Endsley in [9]. Some researchers have attempted to assess SA using psychophysiological and behavioural indicators such as eye-tracking measures [39], EEG measurements [12] or cardiac activity [25]. Koester [21] has proposed SA is a more complex cognitive process functioning at a higher level with other underlying factors, such as stress or mental workload. Thus, an alternative technique for SA assessment would be to measure some of the factors underlying the development of SA using psychophysiological measures.

\subsection{Factors influencing SA}

SA could be affected by stress and mental workload [37] as well as including fatigue, work overload, false ground mindset and poor communication [27]. Stress, mental workload and communication are thus measurable factors that we consider to assess SA in RAS.

2.3.1 Mental workload and situation awareness. Kahneman [19] describes mental workload (MW) as the balance between mental demands imposed by a task and attentional resources allocated to it. The theoretical link between MW and SA has been discussed for several decades. Endsley [8] posits that SA and MW are two independent concepts, while others $[17,23]$ suggest that the two concepts are interdependent, especially when MW is high. Various researchers have tested the relationships that may exist between these two notions $[1,13,18,41]$, and a meta-analysis [40] concluded that the MW-SA relationship varies depending on the context.

2.3.2 Stress, mental workload and situation awareness. Lazarus [22] defines stress as the feeling experienced by an individual when their task's demands exceed the personal resources they are able to mobilize. Stress may be internal (fatigue, worry, etc.) or external (noise, time pressure, etc.). Stress first has an influence on MW. Authors show that increased stress reduces working memory resources, thus increasing MW [20], and that operators are sensitive to distractors when stress and MW are low [41]. Studies show that some physiological indicators are related to both processes, including electrodermal activity (EDA), heart rate (HR), and heart rate variability (HRV), and those vary in the same way with increasing stress and MW.

Authors studying the relationship between stress and SA have observed that high levels of stress can cause a loss of SA. Stressors decrease team performance and awareness of the team's needs [41]. Stress can also reduce an operator's field of attention and thus degrade perceived information, leading to premature decision making [30] Importantly, stressed operators would still believe having good SA, while their mental model of the situation diverges from reality, increasing operational risks. Few studies have examined the relationship between stress and SA in surgery.

2.3.3 Communication and situation awareness. Information exchange between surgical team members is mostly based on communication. Ineffective communication (e.g. delays) can degrade the team SA, leading to reduced performance, increased errors, and adverse events [38]. Communication is a major process for the building and maintaining team SA and for effective teamwork [28].

Randell et al. [32] theorized that more explicit verbal communication occurs in RAS. They described behavioural indicators of communication quality and observed that some could be related to individual SA and team SA. (a) The number of equipment-related questions and oral confirmations, could be related to team SA; (b) low SA may be associated with repetitions, a lack of verbalization of actions, or the emergence of off-topic discussion; (c) high SA was associated with numerous verbalizations of actions, and with team proximity to the operating table; (d) who raises alert when complications occur is also an indicator of SA: if the surgeon alerts of issues, it would indicate low team SA; for other team members, high team SA. Finally, high team SA is related to team coordination patterns, e.g. anticipating the actions of other team members.

\section{MODEL AND HYPOTHESES}

Taking into account the literature presented above, we have developed a model with the aim of explaining the relationship between the concepts of mental workload, stress, and communication with situation awareness (Figure 2). Stress and MW influence each other, presumably varying in a similar way. Stress influences SA, but not the other way around $[30,41]$, potentially because operators tend to be unaware of their SA loss [11,30]. MW influences SA, and this influence may vary depending on the context [40]. Additionally, MW and stress have an influence on team communication quality. Finally, communication influences SA [27].

The main objective of this research is to propose a multimodal method for the assessment of SA in RAS based on the proposed model. This leads us to formulate the following hypotheses which we intend to validate in a study in real surgery environments.

H1 Individual situation awareness can be influenced by the mental workload level on one side, and stress on the other side. 


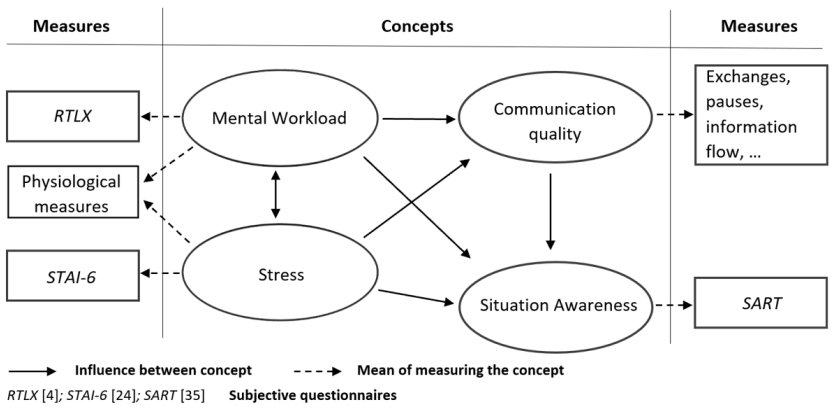

Figure 2: Graphical representation of our SA model.

H2 Individual and team situation awareness are influenced by the quality of communication.

In the following section, we present an ongoing study aimed at validating these hypotheses.

\section{METHOD}

\subsection{Participants}

Participants in this study were members of the surgical team, at a major teaching hospital, performing radical prostatectomy surgery using a Da Vinci Xi Robotic Surgical System. We have recorded two radical prostatectomy operations and are expecting to record at least another four procedures.

\subsection{Materials}

Three questionnaires were used to gather subjective feedback from the surgeons: situation awareness, mental workload and stress. An Empatica E4 sensor was selected to collect surgeons' physiological data due to its simplicity in implementation. In addition, two fixed cameras were positioned in the operating room (OR) to record operators' behaviours, including communication, and movement within the OR. One camera is positioned to record a view of the assistant and circulating nurse at the operation table, and a second camera to have a view on the other parts of the operation room. Two microphones were used: one placed near the surgeon and the other close to the operating table. The surgical view was also recorded. During pre-determined safe moments in the operation, the surgeon pauses the operation to complete the three questionnaires on stress, MW and SA. Physiological and video recordings were uninterrupted throughout the procedure.

4.2.1 Self-rating questionnaires. The three questionnaires administered in this study were the STAI-6, RTLX and SART, which respectively measure stress, MW and SA experienced by operators during the execution of a task. These questionnaires are administered at different points in the surgical procedure to allow a posteriori comparison of these constructs. The STAI-6 [24] is an effective scale for evaluating the stress felt by surgeons when performing surgical tasks [1]. The NASA-TLX is a scale that is sensitive to variations in MW in laparoscopic surgery [44]; here, we used RTLX [4], a short version of the NASA-TLX. We removed some sub-scales less relevant to our context [15], such as the "physical demand" subscale since the RAS console reduces necessary physical resources to perform the task. Finally, the SART [35] measures an operator's subjective SA. This instrument assesses the operator's SA based on queries that do not need to be adapted to the content of the scenario [34].

4.2.2 Physiological measures. Variation of HR, HRV and EDA are influenced by emotions, stress, MW or environmental stimuli [6]. We use those signals as indicators of stress and MW throughout the procedure, collecting them with the wrist-worn Empatica E4 sensor. This watch allows time markers to be recorded. At the beginning and end of each major procedure step, the surgeon briefly presses the Empatica E4 button to record a timestamp, allowing for easy segmentation of the physiological data. HR is collected at a frequency of $1 \mathrm{~Hz}$ and EDA at a frequency of $4 \mathrm{~Hz}$. The surgeon will only be able to wear this device at the console controlling the robotic assisted device, because of the hygiene standards in place in the operating theatre.

4.2.3 Communication and team processes. These team processes are assessed through behavioural analysis, done offline from a video analysis of the surgical situation. The presence, absence and quantity of the indicators described by Randell et al. [32], which allow the evaluation SA quality through communication and team processes, are used to give an indication of operators' SA.

\subsection{Procedure}

The experimenter prepares the rating scales to be administered during the operation, the cameras, microphones, and the E4 sensor and then explains to the surgical team that they will be interrupted at several safe moments during the operation. During the breaks, the surgeon completes the three self-rating questionnaire on stress, MW and SA. Finally, once the surgical procedure is completed, the experimenter stops the audio-visual and Empatica recordings.

\section{DATA ANALYSIS}

In order to realize an analysis of SA in RAS using different types of parameters, a coding scheme was built on the model of a multimodal matrix presented in the study by Echeverria et al. [6]. This document includes parameters describing the surgical activity and various raw physiological values (Figure 3). The first part of the table contains timestamps and the value corresponding to the surgical step being performed. The second part contains the surgical activity coding (cf. 5.3). The third part contains the raw physiological values. Since this document contains only data that can be measured per second, measurements corresponding to time segments (questionnaire scores, higher-level physiological features) are not included. During this video and physiological data coding, we also searched for points of interest that could attest to variations in the operators' situation awareness during the operation.

\subsection{Subjective questionnaires}

The questionnaires were administered at each major procedure step and therefore their scores are computed for each surgical step. These scores will be analysed jointly with the corresponding physiological data. This will make it possible to study the correlations between the different parameters derived from the analysis of the physiological data, and the subjective levels of MW, stress and SA following the completion of a given surgical step. 


\begin{tabular}{|c|c|c|c|c|c|c|c|c|c|c|c|c|c|}
\hline Time & Surg' step & S-talking & ... & S-listening & .. & Verbal Comm' & Comm' type & Non-verbal Comm' & .. & SCL & EDA peak & SCR Amp & HR \\
\hline $00: 00: 21$ & 1 & 1 & $\ldots$ & 0 & $\ldots$ & Action verbalisation & 3 & 1 & $\ldots$ & 4.129 & 1 & 0.09076 & 80 \\
\hline $00: 00: 22$ & 1 & 1 & $\ldots$ & 0 & $\ldots$ & Action verbalisation & 3 & 1 & $\ldots$ & 4.08 & 0 & & 79.9 \\
\hline $00: 00: 23$ & 1 & 0 & $\ldots$ & 1 & $\ldots$ & Question & 3 & 1 & ... & 4.059 & 0 & & 79.75 \\
\hline$\ldots$ & $\ldots$ & $\ldots$ & $\ldots$ & $\ldots$ & $\ldots$ & $\ldots$ & $\ldots$ & $\ldots$ & $\ldots$ & $\ldots$ & $\ldots$ & & $\ldots$ \\
\hline
\end{tabular}

Figure 3: Document built based on the model of a multimodal matrix (Incomplete view of the matrix)

\subsection{Physiological data}

In order to analyse the physiological data (Figure 4), all data streams were synchronized at $1 \mathrm{~Hz}$, applying a filter to data with a higher frequency. Using the Ledalab EDA analysis software, we performed an artifact analysis of the EDA data and a CDA (continuous decomposition analysis) to obtain a file listing the appearance of each skin conductance response (SCR) as well as their amplitude. A peak in the EDA was defined by a minimum increase of $0.03 \mu \mathrm{S}$ as suggested in the literature [3]. Following the collection of these parameters, and for each steps of the procedure, we derive the following parameters: number of significant SCRs, sum of the amplitude of SCRs. The skin conductance level (SCL) was also calculated for each step of the procedure. According to Braithwaite et al. [3], SCL values constantly change within an individual. Consequently, the SCL baseline must be regularly updated to analyse local SCL changes. For this reason, we use the first and last minute of each procedure step to evaluate local changes in the SCL.

Concerning HR and HRV analysis, inter-beat interval data was first cleaned by means of artifact correction using the Kubios HRV software. Time-domain and frequency-domain analysis were performed to obtain the following parameters for each procedure step and for points of interest identified during the video analysis: Mean RR, SDNN, Mean HR, Min and Max HR, RMSSD, NN50, and pNN50 (time-domain); VLF, LF, HF and LF/HF ratio (freq-domain). For the HRV analysis, we analyse the five minutes before the administration of each questionnaire as suggested by the literature [36].

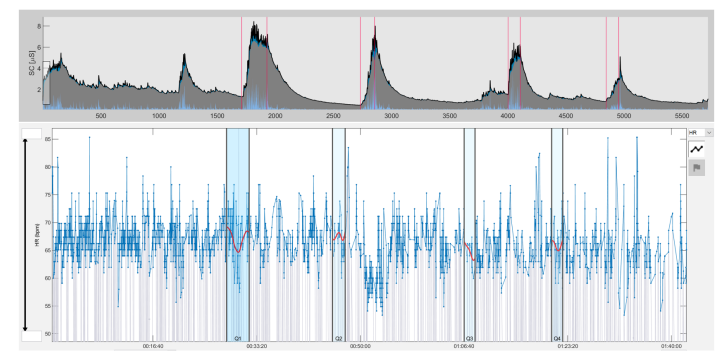

Figure 4: Raw physiological data with markers.

\subsection{Communication and team processes}

A behavioural analysis of communication and team processes is being carried out using videos of the surgical situation. A transcript listing all the communication events between the operators was obtained for each surgery. In a first part, the document specifies which operators are talking and listening. Then, it includes indicators relating to verbal and non-verbal communication. In our study, the indicators collected are those mentioned by Randell et al. [32] as being indicators of the operator and team's levels of situation awareness. Non-verbal communication refers to times when speech is present or absent. This indicator consists of coding the moments when operators communicate or do not communicate.
The analysis of the communication will then consist of coding the presence or absence of these indicators throughout the procedure. An absence will be coded " 0 " and a presence " 1 ". Actions of the operators are coded with specific keywords according to the actions carried out. As in Koester [21], the communication is categorized into three types: actual, relevant and general communication. Actual communication refers to necessary interactions in a given situation; Relevant communication refers to an interaction that is not important in a given situation, but relevant to the safe conduct of the operation; General communication refers to interaction which are neither important nor relevant to the safe conduct of the operation. The column "communication type" was thus coding using a value 1, 2 or 3 for actual, relevant and general communication.

\subsection{Multimodal analysis}

After pre-processing the multimodal data, the next step is an intraand inter-step analysis. This analysis will consist in calculating the number of occurrences of each communication indicator and the averages of the physiological parameters, for each step in the surgical procedure. Physiological measures, STAI-6, RTLX scores and communication indicators will be compared with SA scores (SART) to assess the influence of stress, MW and communication on SA. Observation of correlations could validate our model and demonstrate that the operator's SA can be predicted from communication, physiological signals, stress and MW measurements.

\section{CONCLUSION AND FUTURE WORK}

We presented an exploratory study with the goal of measuring the cognitive state of situation awareness from multimodal data. For this purpose, the aim of this study is to collect preliminary data in order to validate our model, with the hypothesis that operators' situational awareness can be derived from the study of variations in mental workload, stress, and communication within the team.

This study firstly attests to the difficulty of implementing a method for assessing situation awareness in the wild using parameters from multimodal data sources such as physiological, subjective, and behavioural indicators. Indeed, most of the studies evaluating this cognitive state in this area were conducted by observation and/or interview. The objective of this study is thus to try to obtain more objective indicators that can be measured directly. One interest would be to be able to offer operators feedback on their activity and performance and to warn them before the occurrence of potential risk situations, by identifying a drop in individual or collective situation awareness's through the aggregation and in real-time analysis of physiological and behavioural data.

\section{ACKNOWLEDGMENTS}

This work was supported in part by Atlanstic 2020 RFI Chaire Internationale ARTIST. The authors extend thanks to the OR staff at CHU Nantes. 


\section{REFERENCES}

[1] Brittany L Anderson-Montoya and Mark W Scerbo. 2019. Human Factors Psychology in Surgery. In Comprehensive Healthcare Simulation: Surgery and Surgical Subspecialties. Springer, 153-167.

[2] Abdullatif Aydin, Nicholas Raison, Muhammad Shamim Khan, Prokar Dasgupta, and Kamran Ahmed. 2016. Simulation-based training and assessment in urological surgery. Nature Reviews Urology 13, 9 (2016), 503.

[3] Jason J Braithwaite, Derrick G Watson, Robert Jones, and Mickey Rowe. 2013. A guide for analysing electrodermal activity (EDA) \& skin conductance responses (SCRs) for psychological experiments. Psychophysiology 49, 1 (2013)

[4] James C Byers. 1989. Traditional and raw task load index (TLX) correlations: Are paired comparisons necessary? Advances in Industrial Erfonomics and Safety l. Taylor and Francis. (1989).

[5] Nancy J Cooke, R Stout, Eduardo Salas, et al. 2001. A knowledge elicitation approach to the measurement of team situation awareness. New trends in cooperative activities: Understanding system dynamics in complex environments (2001) 114-139.

[6] Roberto et Buckingham Shum Simon Echeverria, Vanessa et Martinez-Maldonado 2019. Vers une collaboration translucide: Donner du sens aux données d'un groupe multimodal. In Actes de la conférence CHI 2019 sur les facteurs humains dans les systèmes informatiques. 1-16.

[7] M.R. Endsley. 1988. Situation awareness global assessment technique (SAGAT) In Proceedings of the IEEE 1988 National Aerospace and Electronics Conference. 789-795 vol.3. https://doi.org/10.1109/NAECON.1988.195097

[8] Mica R Endsley. 1993. Situation awareness and workload- Flip sides of the same coin. In International Symposium on Aviation Psychology, 7 th, Columbus, $\mathrm{OH}$ 906-911.

[9] Mica R Endsley. 1995. Measurement of situation awareness in dynamic systems. Human factors 37, 1 (1995), 65-84.

[10] Mica R. Endsley. 1995. Toward a Theory of Situation Awareness in Dynamic Systems. 37, 1 (1995), 32-64. https://doi.org/10.1518/001872095779049543 Publisher: SAGE Publications Inc.

[11] Mica R. Endsley. 2020. The Divergence of Objective and Subjective Situation Awareness: A Meta-Analysis. 14, 1 (2020), 34-53. https://doi.org/10.1177/ 1555343419874248 Publisher: SAGE Publications.

[12] Raul Fernandez Rojas, Essam Debie, Justin Fidock, Michael Barlow, Kathryn Kasmarik, Sreenatha Anavatti, Matthew Garratt, and Hussein Abbass. 2019. Encephalographic Assessment of Situation Awareness in Teleoperation of HumanSwarm Teaming. In Neural Information Processing (Cham) (Communications in Computer and Information Science), Tom Gedeon, Kok Wai Wong, and Minho Lee (Eds.). Springer International Publishing, 530-539. https://doi.org/10.1007/9783-030-36808-1 58

[13] Maik Friedrich, Maresa Biermann, Patrick Gontar, Marcus Biella, and Klaus Bengler. 2018. The influence of task load on situation awareness and control strategy in the ATC tower environment. 20, 2 (2018), 205-217. https://doi.org/ 10.1007/s10111-018-0464-4

[14] Aimee K. Gardner, Matthew Kosemund, and Joseph Martinez. 2017. Examining the Feasibility and Predictive Validity of the SAGAT Tool to Assess Situation Awareness Among Medical Trainees. 12, 1 (2017), 17-21. https://doi.org/10. 1097/SIH.0000000000000181

[15] Sandra G Hart. 2006. NASA-task load index (NASA-TLX); 20 years later. In Proceedings of the human factors and ergonomics society annual meeting, Vol. 50 Sage publications Sage CA: Los Angeles, CA, 904-908.

[16] Andrew N Healey, Shabnam Undre, Nick Sevdalis, Maria Koutantji, and Charles A Vincent. 2006. The complexity of measuring interprofessional teamwork in the operating theatre. Journal of interprofessional care 20, 5 (2006), 485-495.

[17] Keith C Hendy. 1995. Situation awareness and workload: Birds of a feather? Defence and Civil Institute of Environmental Medicine.

[18] Brittany E Holthausen, Zoe M Becerra, M David Keller, John M Ziriax, and Bruce N Walker. 2019. Performance in Noise: The Relationship Between Workload And Situation Awareness in Navy Tactical Teams. In Proceedings of the Human Factors and Ergonomics Society Annual Meeting, Vol. 63. SAGE Publications Sage CA: Los Angeles, CA, 1585-1589.

[19] Daniel Kahneman and Kahneman Daniel. 1973. Attention and Effort.

[20] Reza Ul Karim. 2012. The Effect of Stress on Task Capacity and Situational Awareness. (2012). https://library.ndsu.edu/ir/handle/10365/26480 Accepted: 2017-09-12T21:02:07Z Publisher: North Dakota State University.

[21] T Koester. 2019. Situation awareness and situation dependent behaviour adjustment in the maritime work domain. In Human-Centered Computing: Cognitive, Social, and Ergonomic Aspects. Vol. 3. \#255.

[22] Richard S. Lazarus. 1990. Theory-Based Stress Measurement. 1, 1 (1990), $3-$ 13. https://doi.org/10.1207/s15327965pli0101_1 Publisher: Routledge _eprint: https://doi.org/10.1207/s15327965pli0101_1.

[23] Yeong Heok Lee, Jeong-Dae Jeon, and Youn-Chul Choi. 2012. Air Traffic Controllers' Situation Awareness and Workload under Dynamic Air Traffic Situations. 51, 3 (2012), 338-352. https://doi.org/10.5325/transportationj.51.3.0338 Publisher: Penn State University Press.
[24] Theresa M. Marteau and Hilary Bekker. 1992. The development of a six-item shortform of the state scale of the Spielberger State-Trait Anxiety Inventory (STAI). 31, 3 (1992), 301-306. https://doi.org/10.1111/j.2044-8260.1992.tb00997.x_eprint: https://onlinelibrary.wiley.com/doi/pdf/10.1111/j.2044-8260.1992.tb00997.x.

[25] Ranjana K. Mehta, S. Camille Peres, Ashley E. Shortz, Wimberly Hoyle, Melissa Lee, Gurtej Saini, Hong-Chih Chan, and Mitchell W. Pryor. 2018. Operator situation awareness and physiological states during offshore well control scenarios. 55 (2018), 332-337. https://doi.org/10.1016/j.jlp.2018.07.010

[26] Lucy Mitchell, Rhona Flin, Steven Yule, Janet Mitchell, Kathy Coutts, and George Youngson. 2013. Development of a behavioural marker system for scrub practitioners' non-technical skills (SPLINTS system). fournal of evaluation in clinical practice 19, 2 (2013), 317-323.

[27] Albert A. Nofi. 2000. Defining and Measuring Shared Situational Awareness. https://apps.dtic.mil/sti/citations/ADA390136 Section: Technical Reports.

[28] Avi Parush, Chelsea Kramer, Tara Foster-Hunt, Kathryn Momtahan, Aren Hunter, and Benjamin Sohmer. 2011. Communication and team situation awareness in the OR: Implications for augmentative information display. 44, 3 (2011), 477-485. https://doi.org/10.1016/j.jbi.2010.04.002

[29] Jason C. Pradarelli, Steven Yule, and Douglas S. Smink. 2020. Evaluating NonTechnical Skills in Surgery. In Health Services Research, Justin B. Dimick and Carrie C. Lubitz (Eds.). Springer International Publishing, 125-135. https://doi. org $/ 10.1007 / 978-3-030-28357-5$ _ 12

[30] Tom Price, Matthew Tenan, James Head, William Maslin, and Michael LaFiandra. 2016. Acute stress causes over confidence in situation awareness. In 2016 IEEE International Multi-Disciplinary Conference on Cognitive Methods in Situation Awareness and Decision Support (CogSIMA). IEEE, 1-6.

[31] Nicholas Raison, Thomas Wood, Oliver Brunckhorst, Takashige Abe, Talisa Ross, Ben Challacombe, Mohammed Shamim Khan, Giacomo Novara, Nicolo Buff, Henk Van Der Poel, et al. 2017. Development and validation of a tool for nontechnical skills evaluation in robotic surgery-the ICARS system. Surgical endoscopy 31, 12 (2017), 5403-5410.

[32] R. Randell, S. A. Honey, J. Hindmarsh, N. Alvarado, J. Greenhalgh, A. Pearman, A. Long, A. Cope, A. Gill, P. Gardner, A. Kotze, D. Wilkinson, D. Jayne, J. Croft, and D. Dowding. 2017. A realist process evaluation of robot-assisted surgery: integration into routine practice and impacts on communication, collaboration and decisionmaking. 5, 20 (2017). https://www.journalslibrary.nihr.ac.uk/hsdr/hsdr05200/\#/ abstract Number: 20 Publisher: National Institute for Health Research.

[33] Eduardo Salas, Carolyn Prince, David P Baker, and Lisa Shrestha. 1995. Situation awareness in team performance: Implications for measurement and training. Human factors 37, 1 (1995), 123-136.

[34] Christian M Schulz, Mica R Endsley, Eberhard F Kochs, Adrian W Gelb, and Klaus J Wagner. 2013. Situation Awareness in AnesthesiaConcept and Research. Anesthesiology: The Journal of the American Society of Anesthesiologists 118, 3 (2013), 729-742.

[35] Stephen J Selcon and RM Taylor. 1990. Evaluation of the Situational Awareness Rating Technique(SART) as a tool for aircrew systems design. AGARD, Situational Awareness in Aerospace Operations 8 p(SEE N 90-28972 23-53) (1990).

[36] Fred Shaffer and JP Ginsberg. 2017. An overview of heart rate variability metrics and norms. Frontiers in public health 5 (2017), 258.

[37] Kip Smith and P. A. Hancock. 1995. Situation Awareness Is Adaptive, Externally Directed Consciousness. 37, 1 (1995), 137-148. https://doi.org/10.1518/ 001872095779049444 Publisher: SAGE Publications Inc.

[38] Renée J Stout, Janis A Cannon-Bowers, Eduardo Salas, and Dana M Milanovich. 1999. Planning, shared mental models, and coordinated performance: An empirical link is established. Human Factors 41, 1 (1999), 61-71.

[39] Geoffrey Tien, M Stella Atkins, Bin Zheng, and Colin Swindells. 2010. Measuring situation awareness of surgeons in laparoscopic training. In Proceedings of the 2010 symposium on eye-tracking research \& applications. 149-152.

[40] Michael A Vidulich. 2000. The relationship between mental workload and situation awareness. In Proceedings of the human factors and ergonomics society annual meeting, Vol. 44. SAGE Publications Sage CA: Los Angeles, CA, 3-460.

[41] Ana Wheelock, Amna Suliman, Rupert Wharton, ED Babu, Louise Hull, Charles Vincent, Nick Sevdalis, and Sonal Arora. 2015. The impact of operating room distractions on stress, workload, and teamwork. Annals of surgery 261, 6 (2015), 1079-1084.

[42] S Yule, Rhona Flin, S Paterson-Brown, N Maran, and D Rowley. 2006. Development of a rating system for surgeons' non-technical skills. Medical education 40, 11 (2006), 1098-1104.

[43] S Yule, A Gupta, D Gazarian, A Geraghty, DS Smink, J Beard, T Sundt, G Youngson, C McIlhenny, and S Paterson-Brown. 2018. Construct and criterion validity testing of the Non-Technical Skills for Surgeons (NOTSS) behaviour assessment tool using videos of simulated operations. British fournal of Surgery 105, 6 (2018), 719-727.

[44] Yuliya Y. Yurko, Mark W. Scerbo, Ajita S. Prabhu, Christina E. Acker, and Dimitrios Stefanidis. 2010. Higher Mental Workload is Associated With Poorer Laparoscopic Performance as Measured by the NASA-TLX Tool. 5, 5 (2010), 267-271. https: //doi.org/10.1097/SIH.0b013e3181e3f329 